\title{
SNHG1 promotes proliferation, migration and invasion of bladder cancer cells via the PI3K/AKT signaling pathway
}

\author{
QUAN DU $^{1}$ and JUAN CHEN ${ }^{2}$ \\ ${ }^{1}$ Department of Urology, Xianyang Central Hospital, Xianyang, Shaanxi 712000; ${ }^{2}$ Department of Urology, \\ Shaanxi Provincial People's Hospital, Xi'an, Shaanxi 710068, P.R. China
}

Received October 11, 2019; Accepted May 15, 2020

DOI: $10.3892 / \mathrm{etm} .2020 .9238$

\begin{abstract}
Long non-coding RNA (lncRNA) small nucleolar RNA host gene 1 (SNHG1) has been previously reported to mediate a number of functions during the progression of cancer. However, its involvement in bladder cancer remain unclear. The aim of the present study was to investigate the expression of SNHG1 in bladder cancer and to identify its potential mechanisms. SNHG1 expression was firstly detected in cancer tissues and cells. The effects of SNHG1 on the malignant phenotypes were then investigated. Furthermore, the influence of SNHG1 on the PI3K/AKT signaling pathway was examined. It was demonstrated that SNHG1 expression was significantly upregulated in bladder cancer tissues and cells. Moreover, the loss-of-function experimental results suggested that knockdown of SNHG1 inhibited bladder cancer cell proliferation, migration and invasion, but increased apoptosis; however, SNHG1 overexpression promoted these processes. Mechanistically, rescue assays identified that SNHG1 activated the PI3K/AKT signaling pathway. Therefore, it was speculated that SNHG1 functioned as a carcinogenic lncRNA in bladder cancer via activation of PI3K/AKT.
\end{abstract}

\section{Introduction}

Bladder cancer is the most common malignancy of the urinary system worldwide, with $>76,960$ new cases and 16,390 mortalities estimated in 2016 (1). Bladder cancer is divided into non-muscle-invasive bladder cancer, characterized by a high recurrence rate $(70 \%)$, and muscle-invasive bladder cancer, which has a $<50 \%$ of 5 -year overall survival according to biological characteristics (2). Thus, it is important to identify the molecular mechanism of cellular proliferation

Correspondence to: Dr Juan Chen, Department of Urology, Shaanxi Provincial People's Hospital, 256 Youyi Xi Road, Xi'an, Shaanxi 710068, P.R. China

E-mail: juanchen12@126.com

Key words: bladder cancer, long non-coding RNA, PI3K/AKT signaling pathway, small nucleolar RNA host gene 1 in bladder cancer to facilitate the development of a novel and more effective therapy.

As a class of non-coding RNA without the capacity of coding proteins, long non-coding RNAs (lncRNA) are $>200$ nucleotides in length $(3,4)$. Previous studies have reported that IncRNA are involved in epigenetic, transcriptional and post-transcriptional modulation in various biological processes, including proliferation, differentiation, migration and apoptosis $(5,6)$. IncRNA small nucleolar RNA host gene 1 (SNHG1) located at 11q12.3 locus, functions as an oncogene in a number of cancer types. For instance, Cui et al (7) revealed that high SNHG1 expression in non-small cell lung cancer (NSCLC) was significantly correlated with larger tumor size, advanced TNM stage, lymph node metastasis and poor overall survival. Moreover, $\mathrm{Hu}$ et al (8) reported that knockdown of SNHG1 suppressed gastric cancer cell proliferation both in vitro and in vivo. Liu et al (9) also found that SNHG1 inhibition significantly inhibited cervical cancer cell proliferation, migration and invasion. However, the relationship between SNHG1 expression and bladder cancer, as well as the underlying molecular mechanisms of the oncogenic functions of SNHG1, remains unknown and require further investigation. Furthermore, identifying the downstream targets of SNHG1 will elucidate its critical role in bladder cancer progression.

The PI3K/AKT signaling pathway regulates multiple biological processes, including cell proliferation and apoptosis (10). In most cases, inappropriate activation of PI3K/AKT is speculated to induce tumor formation (11). Increased SNHG1 expression has also been reported to promote the activity of the PI3K/AKT signaling pathway (12). However, the molecular mechanisms underlying this phenomenon are not fully understood.

The aim of the present study was to elucidate the key functions of SNHG1 in the proliferation, apoptosis, migration and invasion of bladder cancer cells in vitro, in addition to investigating the signaling pathways that are possibly implicated in this process.

\section{Materials and methods}

Tissues. Tumor tissues and homologous adjacent healthy tissues were donated by 60 patients with bladder cancer, who underwent surgery between July 2016 and January 2018 
during their hospitalization in Shaanxi Provincial People's Hospital (Xi'an, China), and were stored at $-80^{\circ} \mathrm{C}$ prior to RNA isolation. The inclusion criteria were as follows: i) Patients diagnosed by pathological biopsies; ii) patients at stage I or II based on the TNM staging system (13); iii) patients with complete medical record; and iv) patients and their families who were willing to participate. The exclusion criteria were as follows: i) Patients who were treated within 3 months before admission; ii) patients who had other diseases, such as chronic diseases and metabolic diseases; and iii) patients who were not willing to donate plasma samples. The 60 patients with bladder cancer included 34 males and 26 females (age range, 27-67 years; mean age, $46.4 \pm 5.1$ years). Informed consent was obtained and all experimental procedures were approved by the Human Ethics Committee of Shaanxi Provincial People's Hospital.

Cell culture, transfection and treatment. In total, four human bladder cancer cell lines (T24, SW780, J82 and RT4) and normal bladder epithelial HCV-29 cells were purchased from the American Type Culture Collection (ATCC). Cells were incubated in DMEM supplemented with $10 \% \mathrm{FBS}, 100 \mathrm{U} / \mathrm{ml}$ penicillin and $100 \mu \mathrm{g} / \mathrm{ml}$ streptomycin (all from Invitrogen; Thermo Fisher Scientific, Inc.) in a humidified atmosphere with $5 \% \mathrm{CO}_{2}$ at $37^{\circ} \mathrm{C}$.

For knockdown of SNHG1 in SW780 cells, the sequences of short hairpin (sh)RNA targeting for SNHG1 (5'-CAGCAG TTGAGGGTTTGCTGTGTAT-3') were designed by Shanghai GenePharma Co., Ltd. For overexpression, the full-length SNHG1 was cloned into a pcDNA3.1 vector (Shanghai GenePharma Co., Ltd.) to overexpress SNHG1 in RT4 cells, and the primer sequences were as follows: SNHG1 forward, 5'-GGGGTACCGTTCTCATTTTTCTACTGCTCGTG-3' and reverse, 5'-CGGGATCCATGTAATCAATCATTTTATTAT TTTCATC-3'. The empty vector and scrambled shRNA for SNGH1 were used as negative controls. Cell transfections were conducted using shRNAs and plasmids, both at a final concentration of $100 \mathrm{nM}$, using Lipofectamine ${ }^{\circledR} 2000$ reagent (Invitrogen; Thermo Fisher Scientific, Inc.) following the manufacturer's instructions. Cells were treated with the PI3K agonist 740Y-P (50 $\mu \mathrm{M}$; Sigma-Aldrich; Merck KGaA) or the PI3K inhibitor LY294002 (50 $\mu \mathrm{M}$; Sigma-Aldrich; Merck $\mathrm{KGaA}$ ) for $24 \mathrm{~h}$ at $37^{\circ} \mathrm{C}, 48 \mathrm{~h}$ after transfection.

Reverse transcription-quantitative PCR (RT-qPCR). Total RNA was extracted from tissues or T24, SW780, J82, RT4, and HCV-29 cells using the TRIzol ${ }^{\circledR}$ Reagent (Thermo Fisher Scientific, Inc.) and then converted into cDNA by reverse transcription using PrimeScript ${ }^{\mathrm{TM}}$ RT Master Mix (cat. no. RR036Q; Takara Biotechnology Co., Ltd.), according to the manufacturer's protocol. qPCR was performed using Path-ID ${ }^{\mathrm{TM}}$ Multiplex One-Step RT-PCR Kit (cat. no. 4442136; Applied Biosystems; Thermo Fisher Scientific, Inc.) according to manufacturer's protocol in an ABI 7500 RT PCR system (Applied Biosystems; Thermo Fisher Scientific, Inc.) to determine the relative quantification of SNHG1 expression. A total of $1 \mu \mathrm{g}$ total RNA was reversely transcribed using oligo(dT) primer at $42^{\circ} \mathrm{C}$ for $1 \mathrm{~h}$ and $2 \mu \mathrm{l}$ the reverse transcription reaction mix was amplified by PCR with denaturation at $95^{\circ} \mathrm{C}$ for $2 \mathrm{~min}$, followed by 50 cycles of $95^{\circ} \mathrm{C}$ for $30 \mathrm{sec}, 55^{\circ} \mathrm{C}$ for
$30 \mathrm{sec}$ and $72^{\circ} \mathrm{C}$ for $1 \mathrm{~min}$. The thermocycling conditions were as follows: Initial denaturation at $95^{\circ} \mathrm{C}$ for $30 \mathrm{sec}$, followed by 40 cycles of $95^{\circ} \mathrm{C}$ for $5 \mathrm{sec}$ and $60^{\circ} \mathrm{C}$ for $30 \mathrm{sec}$. Relative expression was calculated with the $2^{-\Delta \Delta \mathrm{Cq}}$ method as previously described (14), where GAPDH was used as the internal control. The primer sequences were as follows: SNHG1 forward, 5'-AGGCTGAAGTTACAGGTC-3' and reverse, 5'-TTGGCT CCCAGTGTCTTA-3'; and GAPDH forward, 5'-GTCAAC GGATTTGGTCTGTATT-3' and reverse, 5'-AGTCTTCTG GGTGGCAGTGAT-3'.

Cell Counting Kit-8 (CCK-8) assay. Cell proliferation was assessed using a CCK- 8 assay (Beyotime Institute of Biotechnology). At 0, 24, 48, 72 and 96 h post-transfection, cells were re-seeded into culture medium on 96 -well plates at a density of $5 \times 10^{3}$ cells/well and incubated at $37^{\circ} \mathrm{C}$ overnight. CCK-8 solution (Beyotime Institute of Biotechnology) with $10 \mu \mathrm{l} /$ well was then added and incubated at $37^{\circ} \mathrm{C}$ for another $2 \mathrm{~h}$ according to the manufacturer's instruction. Absorbance was measured with a microplate reader at a wavelength of $450 \mathrm{~nm}$.

Colony formation assay. After $24 \mathrm{~h}$ transfection, SW780 and RT4 cells were re-seeded onto 6-well plates at a density of 500 cells per well and cultured for 3 days. After washing with PBS, cells were fixed with $4 \%$ paraformaldehyde solution at $37^{\circ} \mathrm{C}$ for $15 \mathrm{~min}$ and stained with $0.1 \%$ crystal violet solutions at room temperature for $10 \mathrm{~min}$. The number of colonies containing $>50$ cells was analyzed manually using light microscope (magnification, x200). The experiments were performed in triplicate.

Apoptosis detection. For the detection of the percentage of early apoptotic cells, SW780 and RT4 cells were seeded in 24-well plates $\left(2 \times 10^{5}\right.$ cells/well) for $48 \mathrm{~h}$, and stained using Annexin V-FITC apoptosis assay (5 $\mu \mathrm{l}$; Invitrogen; Thermo Fisher Scientific, Inc.) at room temperature for $20 \mathrm{~min}$. The stained cells were analyzed using BD FACSCalibur ${ }^{\mathrm{TM}}$ flow cytometer (Beckman Coulter, Inc.) and FlowJo software (version X; FlowJo LLC). Living cells were in the lower left quadrant. The upper right quadrant represented necrotic and late apoptotic cells, whilst the lower right quadrant represented early apoptotic cells, which were quantified.

Migration assay. Wound-healing assay was performed to measure the cell migration capacity of SW780 and RT4 cells. Transfected cells after $48 \mathrm{~h}$ transfection were subsequently cultured in DMEM in a six-well culture plate at a density of $5 \times 10^{5}$ cells/well. When the confluence reached $95 \%$, the cells were washed and the culture medium was replaced with serum-free DMEM. A scratch was made through the single cell layer using a $10 \mu \mathrm{l}$ pipette tip and the cells were washed again with warmed PBS. After $24 \mathrm{~h}$ incubation in serum-free medium, images of the migrating cells were captured using a light microscope (magnification, x200; Nikon Corporation). Representative images after wounding were captured with a light microscope. The percent closure of the cells into the wound field was measured using ImageJ software (version 1.46r; National Institutes of Health). The rate of wound healing was calculated as: Wound healing 
A

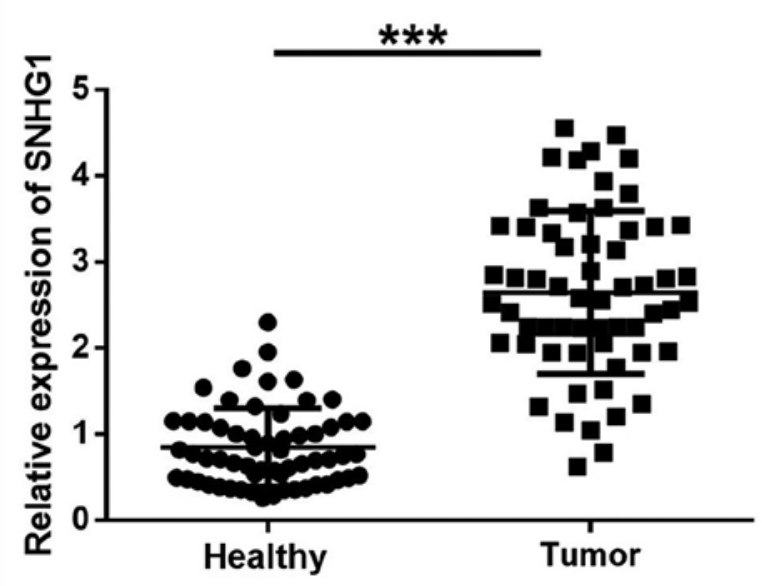

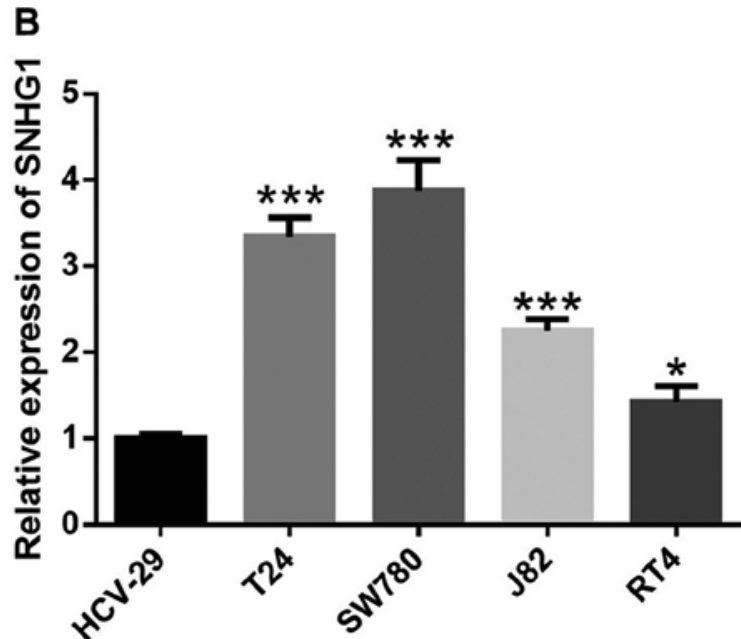

Figure 1.IncRNA SNHG1 expression is significantly upregulated in bladder cancer tissues and cells. (A) SNHG1 expression in 60 paired bladder cancer tissues and adjacent healthy tissues. (B) SNHG1 expression four bladder cancer cell lines, T24, SW780, J82 and RT4 and human immortalized bladder epithelial HCV-29 cells using reverse transcription-quantitative PCR. ${ }^{*} \mathrm{P}<0.05,{ }^{* * *} \mathrm{P}<0.001$ vs.HCV-29 cells or adjacent healthy tissues. SNHG1, small nucleolar RNA host gene 1; lncRNA, long non-coding RNA.

rate $=($ wound width at $0 \mathrm{~h}$-wound width at $24 \mathrm{~h}) /$ wound width at $0 \mathrm{~h} \times 100 \%$.

Transwell assay. Cell invasion was analyzed with Transwell chambers $(8.0-\mu \mathrm{m}$ pore size with polycarbonate membrane; $\mathrm{BD}$ Biosciences) that were pre-coated with Matrigel (BD Biosciences) for $6 \mathrm{~h}$ at $37^{\circ} \mathrm{C}$. Cells resuspended in serum-free medium at a density of $1 \times 10^{4}$ cells/well were added to the upper chamber, while $500 \mu 1$ DMEM with $10 \%$ FBS was added to the bottom chamber. After $24 \mathrm{~h}$, cells that failed to migrate were removed from the upper part of the filters by scrubbing with a cotton swab, and the membrane was fixed with $4 \%$ formaldehyde at room temperature for $5 \mathrm{~min}$ and stained with $0.5 \%$ crystal violet at room temperature for $10 \mathrm{~min}$. The invasive cells were counted using light microscope at x200 magnification from 10 different fields of each filter. The invaded cell rates were calculated using the following formula: Mean test group invaded cell number/mean blank control invaded cell number x $100 \%$.

Western blotting. Total protein from cultured cells were extracted using RIPA buffer (Beyotime institute of Biotechnology). Protein concentration was quantified using a bicinchoninic acid protein assay kit (Thermo Fisher Scientific, Inc.). Equal amounts of protein $(40 \mu \mathrm{g})$ were electrophoresed in a $10 \%$ SDS-PAGE and then electro-blotted onto a PVDF membrane (EMD Millipore). The membranes were incubated with primary antibodies against GAPDH (cat. no. 5174; 1:1,000 dilution; Cell Signaling Technology, Inc.), PI3K (cat. no. 17366; 1:1,000 dilution; Cell Signaling Technology, Inc.), phosphorylated (p)-AKT (cat. no. 4060; 1:1,000 dilution; Cell Signaling Technology, Inc.), AKT (cat. no. 10176-2-AP; 1:1,000 dilution; ProteinTech Group, Inc.), proliferating cell nuclear antigen (PCNA; cat. no. 13110; 1:1,000 dilution; Cell Signaling Technology, Inc.), Bcl-2 (cat. no. 3498; 1:1,000 dilution; Cell Signaling Technology, Inc.), Bax (cat. no. 5023; 1:1,000 dilution; Cell Signaling
Technology, Inc.), N-Cadherin (cat. no. 22018-1-AP; 1:3,000 dilution; ProteinTech Group, Inc.) and E-Cadherin (cat. no. 20874-1-AP; 1:5,000 dilution; ProteinTech Group, Inc.) at $4^{\circ} \mathrm{C}$ overnight. The membranes were then incubated with horseradish peroxidase-conjugated secondary antibodies (cat. no. sc-2004; 1:5,000 dilution; Santa Cruz Biotechnology) for $1 \mathrm{~h}$ at room temperature and visualized using the enhanced chemiluminescence method (Cytiva). GAPDH was used as an internal control. Image-Pro Plus 7.0 software (Media Cybernetics, Inc.) was used to perform the densitometric analysis.

Statistical analysis. SPSS 19.0 software (IBM Corp.) was used to perform statistical analysis. Data are presented as the mean \pm standard deviation from $\leq 3$ independent experiments. Data comparisons were performed by either unpaired (or paired for tissue samples) Student's t-test (between two groups) or one-way ANOVA followed by Tukey's test (among three groups). $\mathrm{P}<0.05$ was considered to indicate a statistically significant difference.

\section{Results}

lncRNA SNHG1 expression is significantly upregulated in bladder cancer tissues and cells. The expression of SNHG1 in bladder cancer tissues was significantly higher compared with the adjacent healthy tissues (Fig. 1A). Moreover, SNHG1 expression was significantly increased in four bladder cancer cell lines, T24, SW780, J82 and RT4 compared with human immortalized bladder epithelial HCV-29 cells (Fig. 1B).

lncRNA SNHG1 promotes bladder cancer cell proliferation. To investigate the association between SNHG1 expression and the proliferative ability of bladder cancer cells, pcDNA-SNHG1, sh-SNHG1 and the empty plasmids were transfected into bladder cancer cells. Transfection with sh-SNHG1 significantly downregulated SNHG1 expression in SW780 cells, 
A

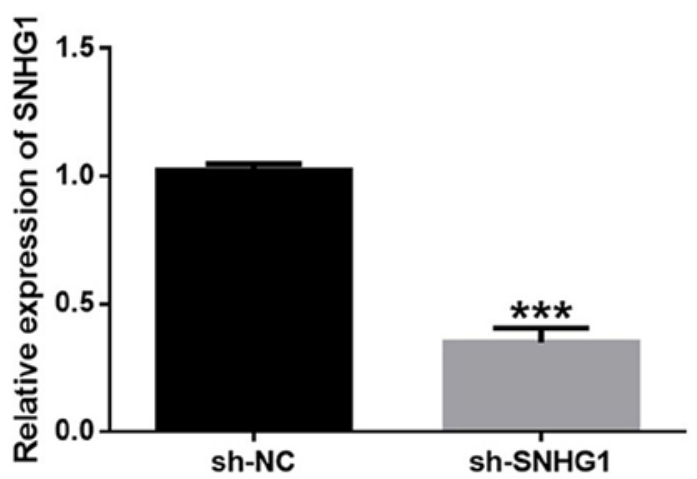

B

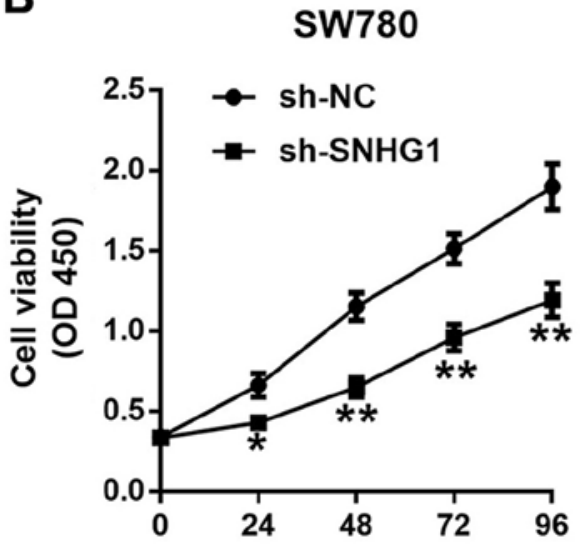

C
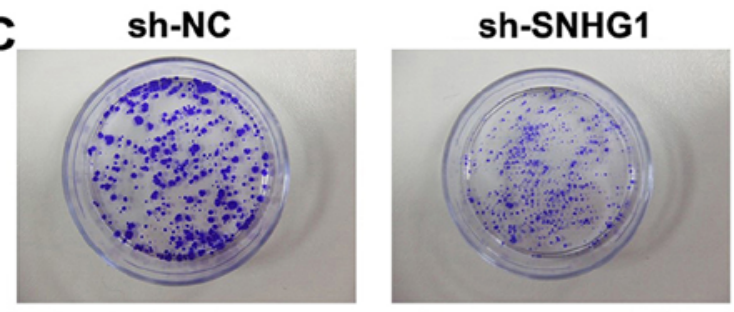

SW780

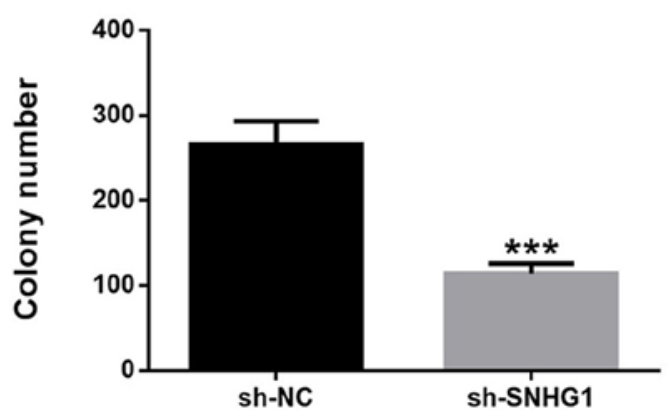

RT4

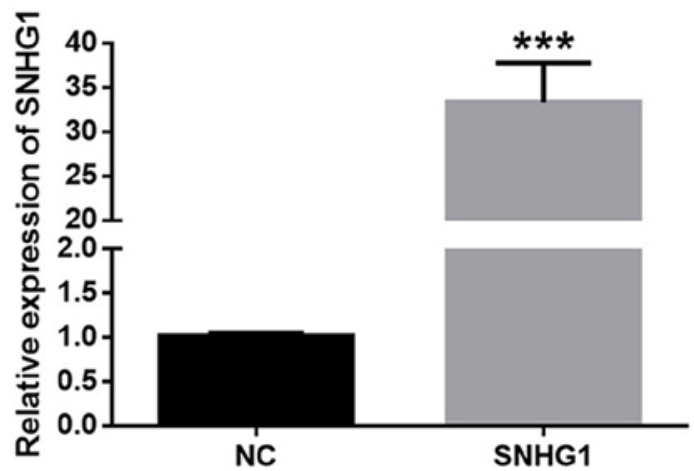

RT4

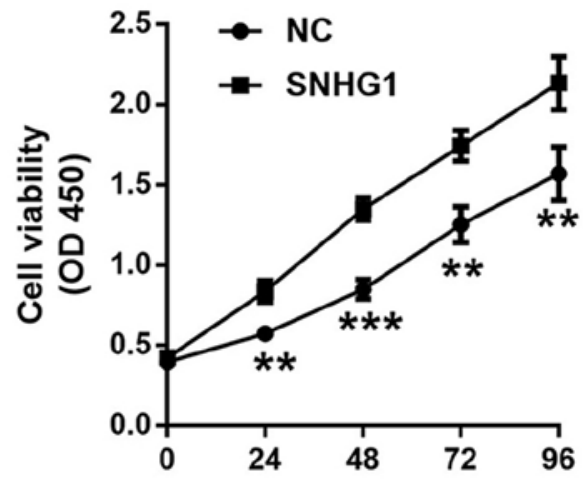

NC

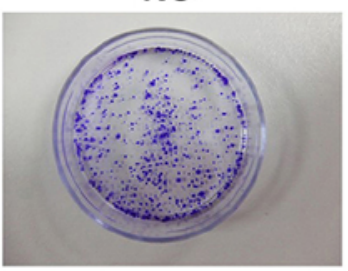

SNHG1

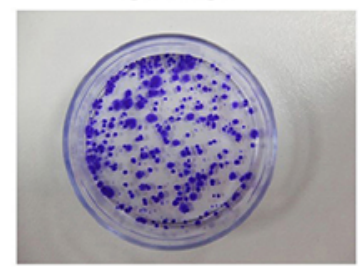

RT4

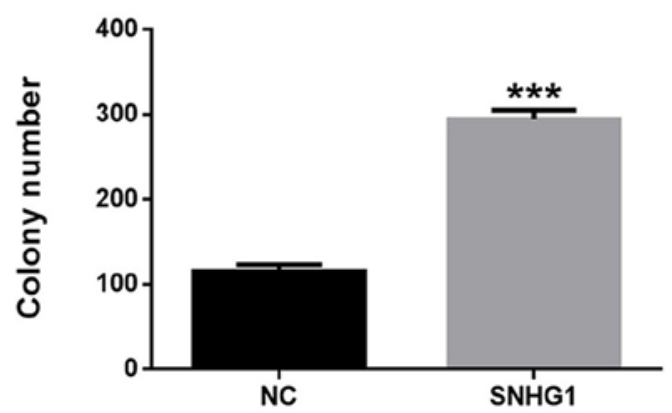

Figure 2. IncRNA SNHG1 promotes bladder cancer cell proliferation. (A) SNHG1 expression, (B) Cell Counting Kit-8 cell viability assay and (C) colony formation assay results in SW780 cells transfected with sh-SNHG1, and in RT4 cells transfected with pcDNA-SNHG1. ${ }^{*} \mathrm{P}<0.05,{ }^{* * *} \mathrm{P}<0.01,{ }^{* * * *} \mathrm{P}<0.001 \mathrm{vs}$. sh-NC or NC. NC, negative control; sh, short hairpin RNA; SNHG1, small nucleolar RNA host gene 1; OD, optical density; lncRNA, long non-coding RNA.

while pcDNA-SNHG1 transfection significantly promoted SNHG1 expression in RT4 cells (Fig. 2A).

The potential biological effects of SNHG1 on the proliferation of bladder cancer cells were detected by CCK- 8 assay and colony formation assay. It was demonstrated that
SNHG1 silencing significantly inhibited the proliferation of SW780 cells, while SNHG1 overexpression led to the opposite results (Fig. 2B and C). These findings indicated that SNHG1 facilitated the proliferation and enhanced the viability of bladder cancer cells. 
A

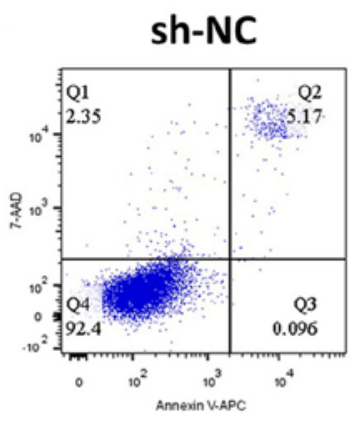

SW780

sh-SNHG1

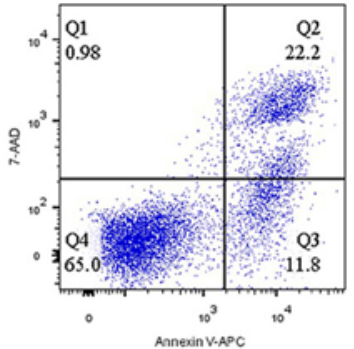

SW780

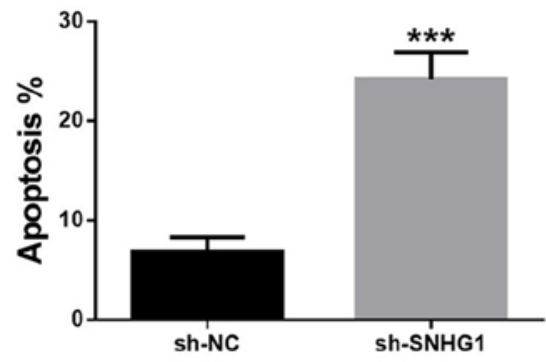

B
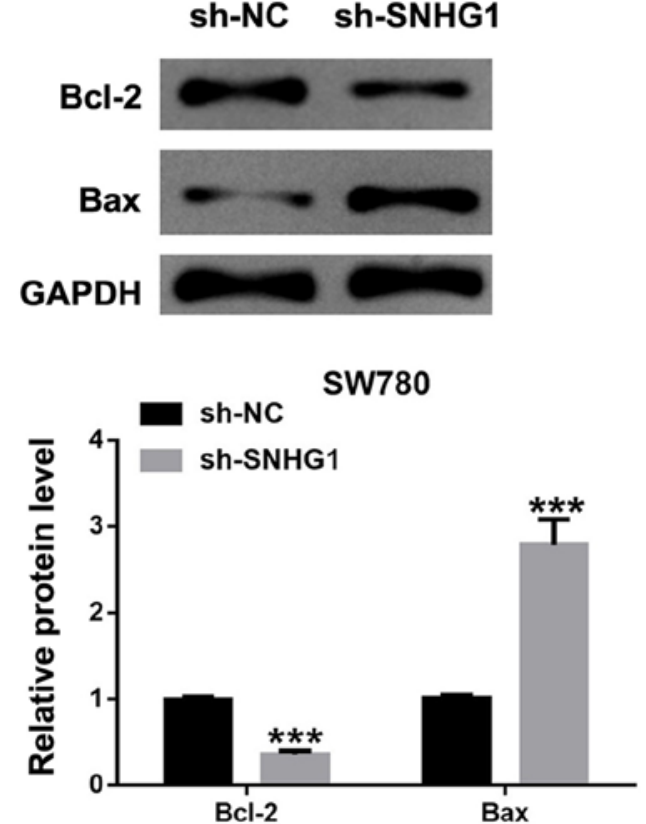

RT4
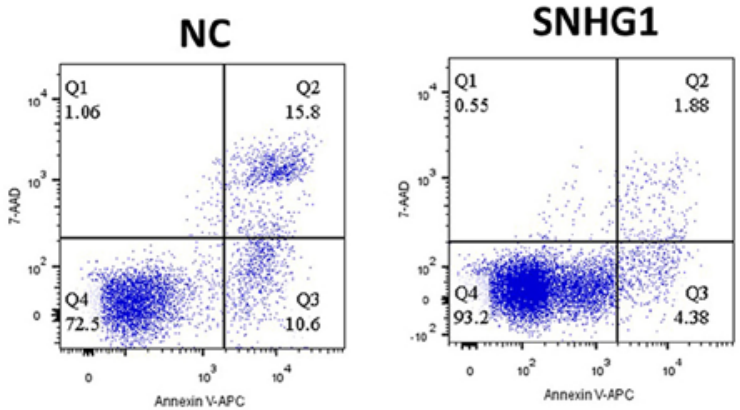

RT4
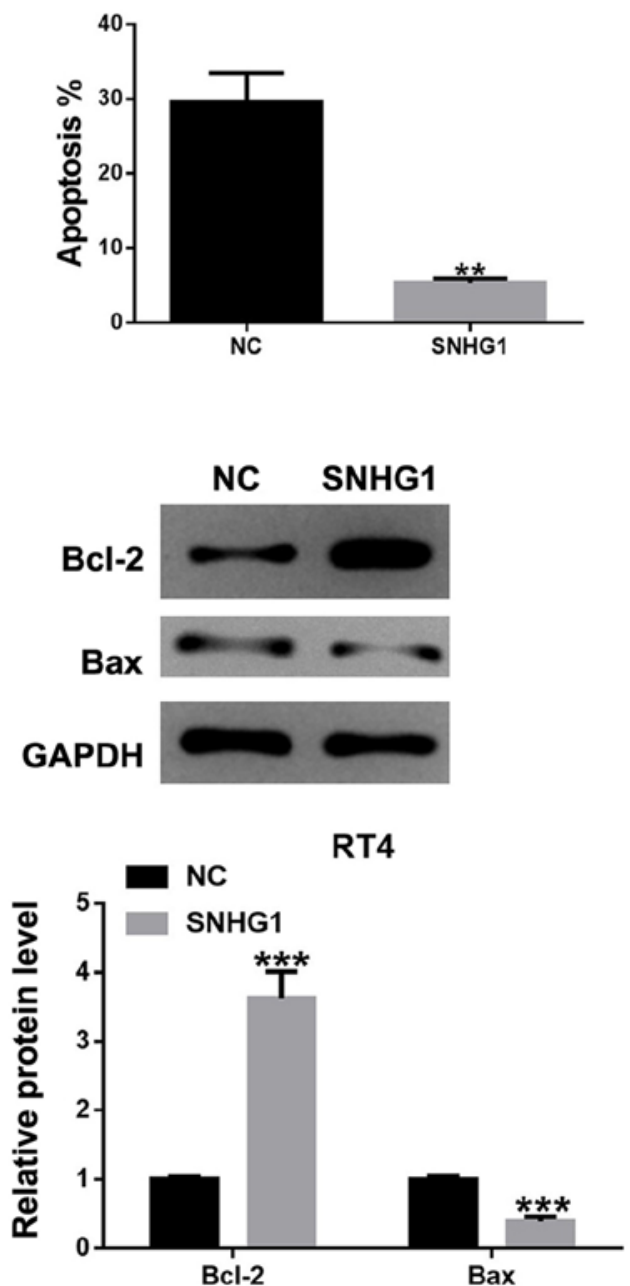

Figure 3. IncRNA SNHG1 inhibits bladder cancer cell apoptosis. SW780 cells were transfected with sh-SNHG1 and in RT4 cells were transfected with pcDNA-SNHG1 (A) Percentage of apoptotic cells using flow cytometry. (B) Protein expression levels of Bcl-2 and Bax were measured using western blotting. ${ }^{* *} \mathrm{P}<0.01,{ }^{* * *} \mathrm{P}<0.001$ vs. sh-NC or NC. NC, negative control; sh, short hairpin RNA; SNHG1, small nucleolar RNA host gene 1; IncRNA, long non-coding RNA.

lncRNA SNHG1 inhibits bladder cancer cell apoptosis. Cell apoptosis analysis results indicated that the number of apoptosis cells was significantly increased in SW780 cells after SNHG1 silencing, while SNHG1 overexpression repressed the number of apoptotic cells (Fig. 3A). Furthermore, the western blotting results demonstrated that the ratio of /Bcl-2/Bax was significantly reduced after SNHG1 knockdown in SW780 cells, while SNHG1 overexpression in RT4 cells led to the opposite effect (Fig. 3B), which was in line with the aforementioned flow cytometric analysis results.

IncRNA SNHG1 increases bladder cancer cell migration and invasion. The suppression of SNHG1 expression decreased the migratory capacity of SW780 cells, while the migratory capacity of RT4 cells was increased in SNHG1-overexpressing cells (Fig. 4A). In addition, the Transwell invasion assay 

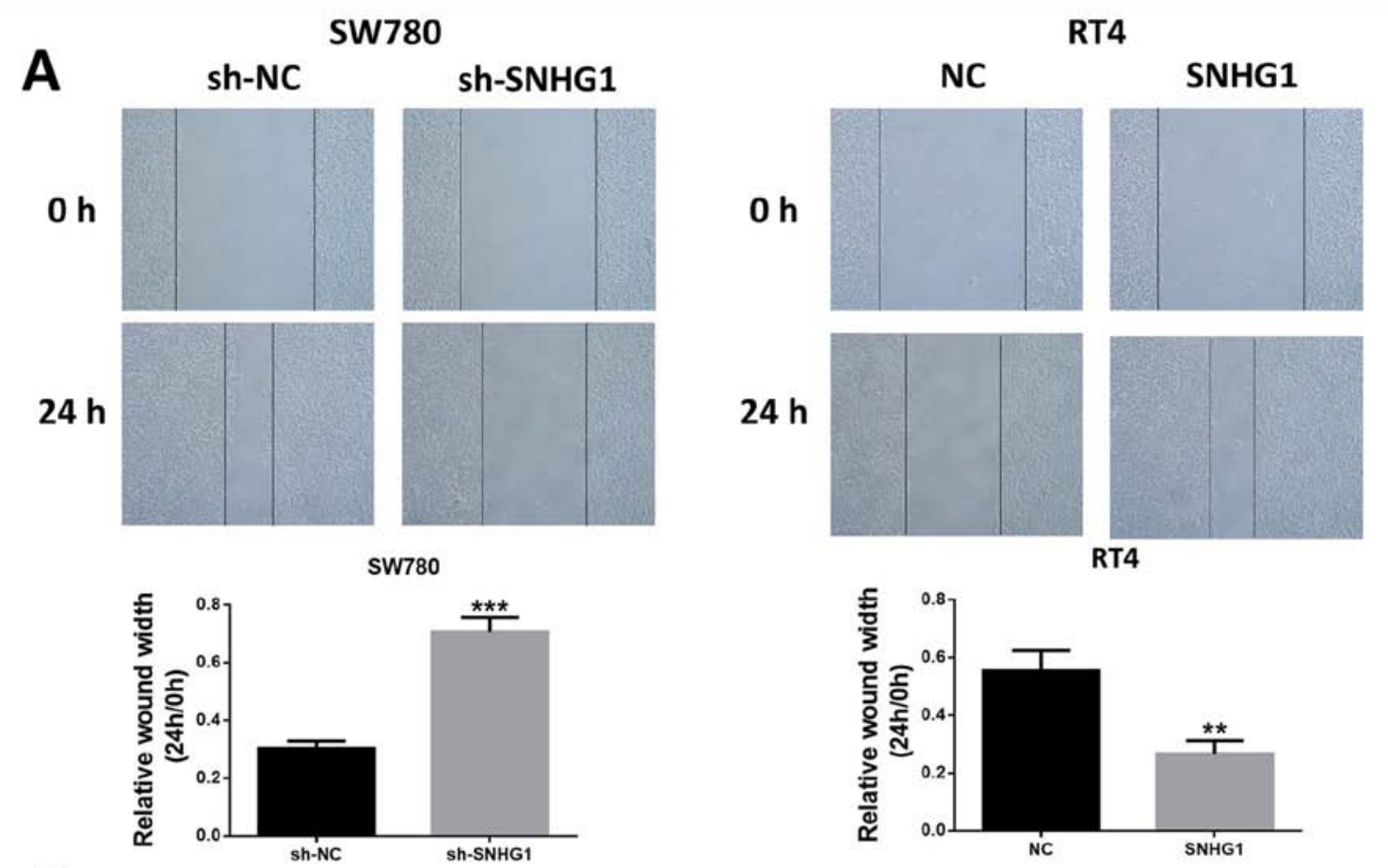

B
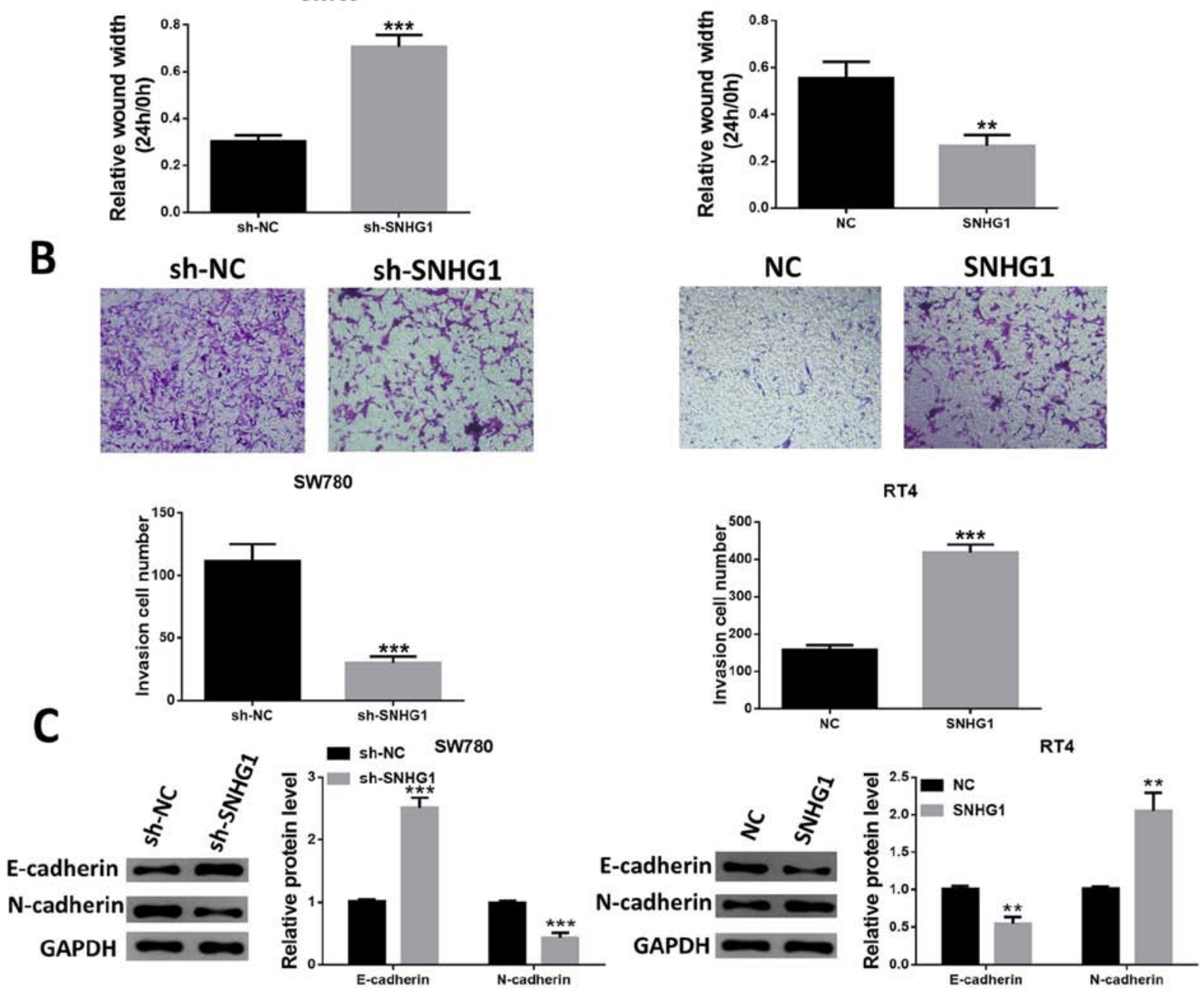

Figure 4. IncRNA SNHG1 elevates bladder cancer cell migration and invasion. SW780 cells were transfected with sh-SNHG1 and RT4 cells were transfected with pcDNA-SNHG1. Cell migration and invasion capacities determined by (A) wound healing and (B) Transwell assay. Magnification, x200. (C) Protein expression levels of E-cadherin and N-cadherin. ${ }^{* *} \mathrm{P}<0.01,{ }^{* * *} \mathrm{P}<0.001$ vs. sh-NC or NC. NC, negative control; sh, short hairpin RNA; SNHG1, small nucleolar RNA host gene 1; lncRNA, long non-coding RNA.

identified similar results, indicating that SW780 cells with SNHG1 knockdown had reduced cell invasion, while RT4 cells with SNHG1 overexpression exhibited enhanced invasive ability (Fig. 4B).

The expression level changes of the epithelial-mesenchymal transition markers E-cadherin and $\mathrm{N}$-cadherin were also examined. Silencing of SNHG1 upregulated E-cadherin expression, but resulted in $\mathrm{N}$-cadherin downregulation.
Furthermore, the expression of E-cadherin was significantly reduced, while the expression of $\mathrm{N}$-cadherin was upregulated following the overexpression of SNHG1 in RT4 cells (Fig. 4C).

PI3K/AKT axis affects the carcinogenesis of SNHG1. The addition of 740Y-P, a PI3K activator, reversed the downregulation of PI3K and AKT phosphorylation levels and the 
A
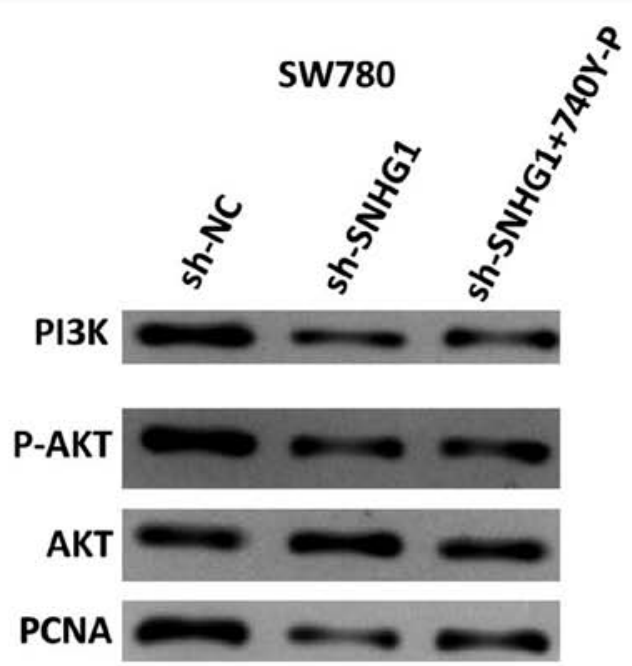

Bcl-2

Bax
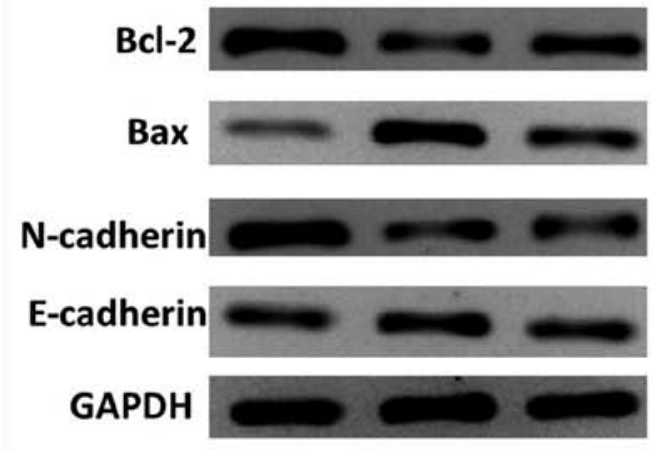

SW780

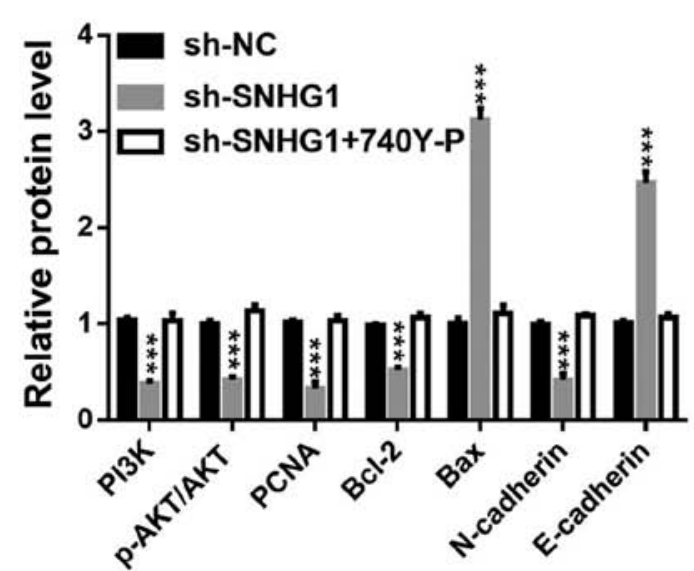

B

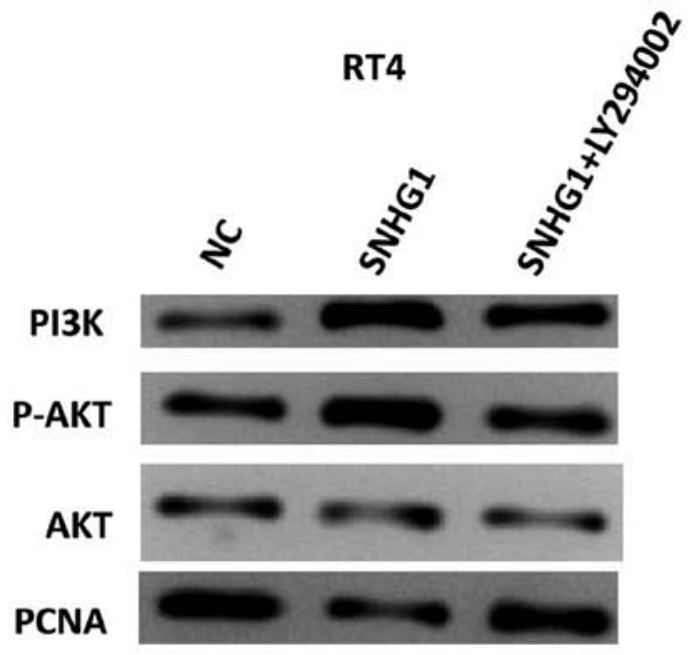

Bcl-2

Bax
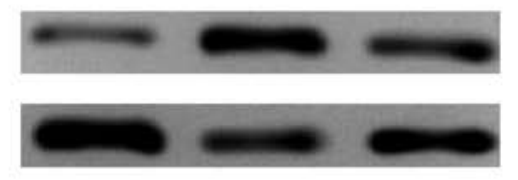

\section{$\mathrm{N}$-cadherin}

E-cadherin

GAPDH
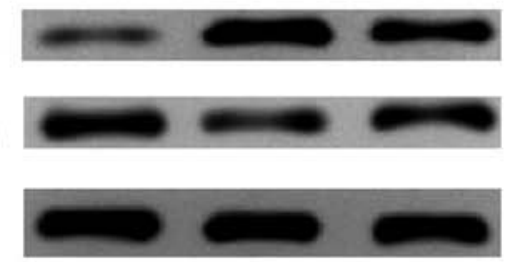

RT4

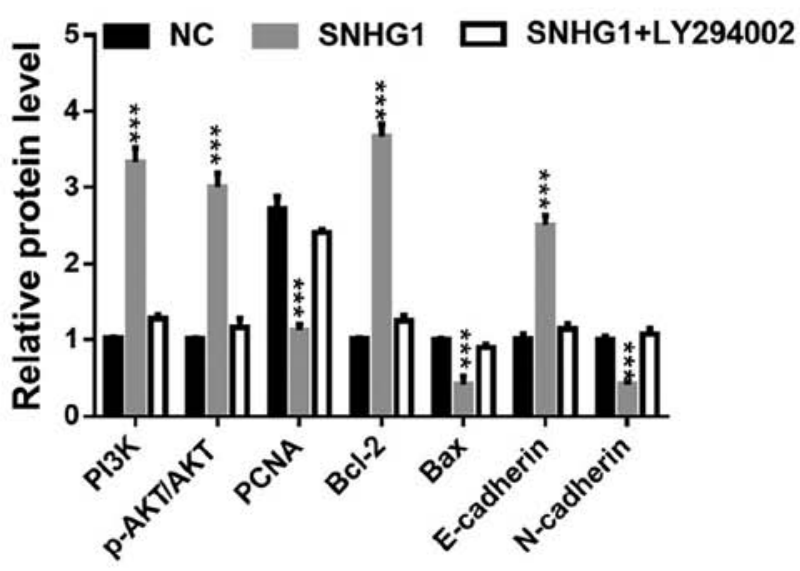

Figure 5. PI3K/AKT axis affects the carcinogenesis of SNHG1. The phosphorylation levels of PI3K and AKT, the protein expression levels of AKT, PCNA, Bcl-2, Bax, N-cadherin and E-cadherin in (A) SW780 cells transfected with sh-SNHG1 + PI3K activator 740Y-P, and (B) in RT4 cells transfected with pcDNA-SNHG1 + PI3K inhibitor LY294002. ${ }^{* * * *} \mathrm{P}<0.001$ vs. sh-NC or NC. NC, negative control; sh, short hairpin RNA; SNHG1, small nucleolar RNA host gene 1; p-, phosphorylated; PCNA, proliferating cell nuclear antigen.

expression levels of PCNA, Bcl-2 and N-cadherin, which were caused by silenced SNHG1. However, 740Y-P reduced the enhanced Bax and E-cadherin protein expression levels induced by silenced SNHG1 (Fig. 5A).

The application of LY294002, a PI3K inhibitor, led to a partial abrogation of SNHG1 overexpression-induced PI3K and AKT phosphorylation, Bcl-2 and E-cadherin upregulation, and PCNA, Bax and N-cadherin downregulation (Fig. 5B).

\section{Discussion}

The initiation and development of bladder cancer involves multiple molecular mechanisms, including abnormal expression of growth factors, adhesion molecules and angiogenic factors (15-17) Therefore, it is important to identify novel biomarker that may be useful for tumor prevention and therapy. In the present study, upregulated SNHG1 expression was identified in bladder cancer tissues and cells. Furthermore, SNHG1 
knockdown significantly suppressed bladder cancer cell proliferation, migration and invasion, as well as promoted apoptosis. However, the overexpression of SNHG1 led to an opposite effect via activation of the PI3K/AKT signaling pathway.

lncRNAs are associated with tumor growth and metastatic potentials in several types of cancer, including bladder cancer. For example, Pei et al (18) reported that lncRNA cancer susceptibility candidate 2 via the inhibition of the Wnt/ $\beta$-catenin signaling pathway, inhibited bladder cancer cell proliferation, migration and invasion, but promoted apoptosis. Wang et al (19) also showed that high expression of lncRNA OIP5 antisense RNA 1 (OIP5-AS1) was a poor predictor of bladder cancer prognosis, and the knockdown of OIP5-AS1 expression decreased cell viability, as well as promoted cell-cycle arrest and apoptosis in bladder cancer. Moreover, Gao et al (20) revealed that lncRNA zinc finger E-box binding homeobox 2 AS1 increased the proliferation, migration and invasion, but reduced the apoptosis of bladder cancer cells as competing endogenous RNA sponges microRNA (miR)-200b to elevate the expression of fascin-1. Previous studies have also reported that SNHG1 serves important roles in the progression of cancer types, including NSCLC (21), glioma (9), pancreatic cancer (22), cholangiocarcinoma (23) and osteosarcoma (24).

Tumor progression is associated with the expression or modulation of several gene products that control apoptosis and proliferation. Apoptosis is an important negative growth regulatory mechanism in tumors (25). In some malignancies, the apoptotic index may reflect the degree of carcinogenicity (26). Bcl-2 is a potent inhibitor of apoptosis and increases proliferation (27). Furthermore, the Bcl-2/Bax ratio is the critical determinant for the induction or inhibition of apoptosis (28). PCNA is present in nuclei throughout the cell cycle and is synthesized in the late $G_{1}$ and $S$ phases (29). Cell migration is a process that is essential during embryonic development, throughout adult life and in some pathological conditions (30). Cadherins, specifically the neural cell adhesion molecule $\mathrm{N}$-cadherin, play an important role in migration (31). In cancer, cadherins control the balance between suppression and promotion of invasion (32). For example, E-cadherin functions as an invasion suppressor and is downregulated in most carcinomas, while $\mathrm{N}$-cadherin, as an invasion promoter, is frequently upregulated (33). The present results suggested that lncRNA SNHG1 was significantly upregulated in bladder cancer tissues and cells. Moreover, shRNA-mediated SNHG1 downregulation impaired cell proliferation, migration and invasion, but facilitated cell apoptosis; however, SNHG1 overexpression led to opposite results.

The PI3K/AKT pathway has been reported to exert important roles in regulating cell cycle and the proliferative, antiapoptotic, metastatic and invasive abilities of cancer cells. For example, the overexpression of lncRNA AB073614 significantly improved the proliferation, migration and invasion of colorectal cancer cells, and decreased the rates of apoptosis and $\mathrm{G}_{1}$ phase cell cycle arrest by targeting the PI3K/AKT signaling pathway (34). It has also been revealed that miR-802 expression inhibited NSCLC tumor growth by deactivating the PI3K/AKT/mTOR pathway by targeting fibroblast growth factor receptor 1 (35). In addition, Zhang et al (36) demonstrated that laminin subunit $\beta-3$ promoted pancreatic ductal adenocarcinoma (PDAC) cell cycle progression, proliferation, invasion and migration, as well as inhibited apoptosis by upregulating the PI3K/AKT signaling pathway. A previous study reported also that SNHG1 acted as an oncogenic lncRNA, and promoted tumorigenesis in PDAC via the PI3K/AKT signaling pathway (12). The present study investigated whether SNHG1 modulated the PI3K/AKT pathway in bladder cancer cells. It was demonstrated that SNHG1 silencing reduced the phosphorylation levels of PI3K and AKT, whilst SNHG1 overexpression induced the activation of the PI3K/AKT axis. The PI3K activator 740Y-P and the inhibitor LY294002 reversed the previous effects of SNHG1 knockdown in SW780 cells and SNHG1 overexpression in RT4 cells, respectively. Based on these findings, it was speculated that SNHG1 may promote the tumorigenic process, at least partly via activating the PI3K/AKT pathway in bladder cancer.

However, several limitations should considered when interpreting the present results. For instance, the number of patients was limited and in vivo experiments were not performed. Furthermore, the other molecular mechanisms that may be involved require further investigation.

\section{Acknowledgements}

Not applicable.

\section{Funding}

No funding was received.

\section{Availability of data and materials}

The datasets used and/or analyzed during the current study are available from the corresponding author on reasonable request.

\section{Authors' contributions}

QD conducted the majority of the experiments, wrote the manuscript and analyzed the data. JC designed the study and revised the manuscript. Both authors read and approved the final manuscript.

\section{Ethics approval and consent to participate}

All experimental procedures were approved by the Human Ethics Committee of Shaanxi Provincial People's Hospital (Xi'an, China).

\section{Patient consent for publication}

Not applicable.

\section{Competing interests}

The authors declare that they have no competing interests.

\section{References}

1. Siegel RL, Miller KD and Jemal A: Cancer statistics, 2016. CA Cancer J Clin 66: 7-30, 2016.

2. Ploussard G, Shariat SF, Dragomir A, Kluth LA, Xylinas E, Masson-Lecomte A, Rieken M, Rink M, Matsumoto K, Kikuchi E, et al: Conditional survival after radical cystectomy for bladder cancer: Evidence for a patient changing risk profile over time. Eur Urol 66: 361-370, 2014 
3. Batista PJ and Chang HY: Long noncoding RNAs: Cellular address codes in development and disease. Cell 152: 1298-1307, 2013.

4. Ling H, Fabbri $M$ and Calin GA: MicroRNAs and other non-coding RNAs as targets for anticancer drug development. Nat Rev Drug Discov 12: 847-865, 2013.

5. Dykes IM and Emanueli C: Transcriptional and Posttranscriptional Gene Regulation by Long Non-coding RNA. Genomics Proteomics Bioinformatics 15: 177-186, 2017.

6. Carlson HL, Quinn JJ, Yang YW, Thornburg CK, Chang HY and Stadler HS: lncRNA-HIT functions as an epigenetic regulator of chondrogenesis through its recruitment of p100/CBP Complexes. PLoS Genet 11: e1005680, 2015.

7. Cui Y, Zhang F, Zhu C, Geng L, Tian T and Liu H: Upregulated lncRNA SNHG1 contributes to progression of non-small cell lung cancer through inhibition of miR-101-3p and activation of Wnt/ $\beta$-catenin signaling pathway. Oncotarget 8: 17785-17794, 2017.

8. Hu Y, Ma Z, He Y, Liu W, Su Y and Tang Z: lncRNA-SNHG1 contributes to gastric cancer cell proliferation by regulating DNMT1. Biochem Biophys Res Commun 491: 926-931, 2017.

9. Liu Y, Yang Y, Li L, Liu Y, Geng P, Li G and Song H: lncRNA SNHG1 enhances cell proliferation, migration, and invasion in cervical cancer. Biochem Cell Biol 96: 38-43, 2018.

10. Chen Y, Wang T, Du J,Li Y, Wang X, Zhou Y, Yu X, Fan W, Zhu Q, Tong X and Wang Y: The Critical Role of PTEN/PI3K/AKT signaling pathway in shikonin-induced apoptosis and proliferation inhibition of chronic myeloid leukemia. Cell Physiol Biochem 47: 981-993, 2018.

11. Singh S, Asal R and Bhagat S: Multifunctional antioxidant nanoliposome-mediated delivery of PTEN plasmids restore the expression of tumor suppressor protein and induce apoptosis in prostate cancer cells. J Biomed Mater Res A 106: 3152-3164, 2018.

12. Zhang Y, Zhang R, Luo G and Ai K: Long noncoding RNA SNHG1 promotes cell proliferation through PI3K/AKT signaling pathway in pancreatic ductal adenocarcinoma. J Cancer 9: 2713-2722, 2018

13. Ward JF and Margulis V: Continous improvement of TNM staging system for bladder cancer. Cancer 115: 704-705, 2009.

14. Livak KJ and Schmittgen TD: Analysis of relative gene expression data using real-time quantitative PCR and the 2(-Delta Delta C(T)) method. Methods 25: 402-408, 2001

15. Poyet C, Thomas L, Benoit TM, Delmo DA, Luberto L, Banzola I, Günthart MS, Sais G, Eberli D, Sulser T and Provenzano M: Implication of vascular endothelial growth factor $\mathrm{A}$ and $\mathrm{C}$ in revealing diagnostic lymphangiogenic markers in node-positive bladder cancer. Oncotarget 8: 21871-2183, 2017.

16. Annels NE, Arif M, Simpson GR, Denyer M, Moller-Levet C Mansfield D, Butler R, Shafren D, Au G, Knowles M, et al: Oncolytic immunotherapy for bladder cancer using Coxsackie A21 virus. Mol Ther Oncolytics 9: 1-12, 2018.

17. Gao Y, Wu K, Chen Y, Zhou J, Du C, Shi Q, Xu S, Jia J, Tang X, $\mathrm{Li} F$, et al: Beyond proliferation: KLF5 promotes angiogenesis of bladder cancer through directly regulating VEGFA transcription. Oncotarget 6: 43791-43805, 2015.

18. Pei Z, Du X, Song Y, Fan L, Li F, Gao Y, Wu R, Chen Y, Li W, Zhou H, et al: Down-regulation of lncRNA CASC2 promotes cell proliferation and metastasis of bladder cancer by activation of the Wnt/ $\beta$-catenin signaling pathway. Oncotarget 8: 18145-18153, 2017.

19. Wang Y, Shi F, Xia Y and Zhao H: lncRNA OIP5-AS1 predicts poor prognosis and regulates cell proliferation and apoptosis in bladder cancer. J Cell Biochem: Nov 28, 2018 (Epub ahead of print). doi: $10.1002 / j \mathrm{cb} .28024$

20. Gao R, Zhang N, Yang J, Zhu Y, Zhang Z, Wang J, Xu X, Li Z, Liu X, Li Z, et al: Long non-coding RNA ZEB1-AS1 regulates $\mathrm{miR}-200 \mathrm{~b} / \mathrm{FSCN} 1$ signaling and enhances migration and invasion induced by TGF- $\beta 1$ in bladder cancer cells. J Exp Clin Cancer Res 38: 111, 2019.
21. Lu Q, Shan S, Li Y, Zhu D, Jin W and Ren T: Long noncoding RNA SNHG1 promotes non-small cell lung cancer progression by up-regulating MTDH via sponging miR-145-5p. FASEB J 32: 3957-3967, 2018

22. Cui L, Dong Y, Wang X, Zhao X, Kong C, Liu Y, Jiang X and Zhang $X$ : Downregulation of long noncoding RNA SNHG1 inhibits cell proliferation, metastasis, and invasion by suppressing the Notch-1 signaling pathway in pancreatic cancer. J Cell Biochem 120: 6106-6112, 2019.

23. Li Z, Li X, Du X, Zhang H, Wu Z, Ren K and Han X: The Interaction Between lncRNA SNHG1 and miR-140 in regulating growth and tumorigenesis via the TLR4/NF- $\mathrm{BB}$ pathway in cholangiocarcinoma. Oncol Res 27: 663-672, 2019.

24. Deng R, Zhang J and Chen J: lncRNA SNHG1 negatively regulates miRNA1013p to enhance the expression of ROCK1 and promote cell proliferation, migration and invasion in osteosarcoma. Int J Mol Med 43: 1157-1166, 2019.

25. Pistritto G, Trisciuoglio D, Ceci C, Garufi A and D'Orazi G: Apoptosis as anticancer mechanism: Function and dysfunction of its modulators and targeted therapeutic strategies. Aging (Albany NY) 8: 603-619, 2016.

26. Gan H, Zhang Y, Zhou Q, Zheng L, Xie X, Veeraraghavan VP and Mohan SK: Zingerone induced caspase-dependent apoptosis in MCF-7 cells and prevents 7,12-dimethylbenz(a) anthracene-induced mammary carcinogenesis in experimental rats. J Biochem Mol Toxicol 33: e22387, 2019.

27. Warren CFA, Wong-Brown MW and Bowden NA: BCL-2 family isoforms in apoptosis and cancer. Cell Death Dis 10: 177, 2019.

28. Mirakhor Samani S, Ezazi Bojnordi T, Zarghampour M, Merat S and Fouladi DF: Expression of p53, Bcl-2 and Bax in endometrial carcinoma, endometrial hyperplasia and normal endometrium: A histopathological study. J Obstet Gynaecol 38: 999-1004, 2018.

29. Tjalma WA, Weyler JJ, Bogers JJ, Pollefliet C, Baay M, Goovaerts GC, Vermorken JB, van Dam PA, van Marck EA and Buytaert PM: The importance of biological factors (bcl-2, bax, p53, PCNA, MI, HPV and angiogenesis) in invasive cervical cancer. Eur J Obstet Gynecol Reprod Biol 97: 223-230, 2001.

30. Paluch EK, Aspalter IM and Sixt M: Focal adhesion-independent cell migration. Annu Rev Cell Dev Biol 32: 469-490, 2016.

31. Cao ZQ, Wang Z and Leng P: Aberrant N-cadherin expression in cancer. Biomed Pharmacother 118: 109320, 2019.

32. Siret C, Terciolo C, Dobric A, Habib MC, Germain S, Bonnier R, Lombardo D, Rigot V and André F: Interplay between cadherins and $\alpha 2 \beta 1$ integrin differentially regulates melanoma cell invasion. Br J Cancer 113: 1445-1453, 2015.

33. Derycke LD and Bracke ME: N-cadherin in the spotlight of cell-cell adhesion, differentiation, embryogenesis, invasion and signalling. Int J Dev Biol 48: 463-476, 2004.

34. Wang Y, Kuang H, Xue J, Liao L, Yin F and Zhou X: IncRNA AB073614 regulates proliferation and metastasis of colorectal cancer cells via the PI3K/AKT signaling pathway. Biomed Pharmacother 93: 1230-1237, 2017.

35. Zhang J, Li J, Li S, Zhou C, Qin Y and Li X: miR802 inhibits the aggressive behaviors of nonsmall cell lung cancer cells by directly targeting FGFR1. Int J Oncol 54: 2211-2222, 12019.

36. Zhang H, Pan YZ, Cheung M, Cao M, Yu C, Chen L, Zhan L, He ZW and Sun CY: LAMB3 mediates apoptotic, proliferative, invasive, and metastatic behaviors in pancreatic cancer by regulating the PI3K/Akt signaling pathway. Cell Death Dis 10: 230, 2019 .

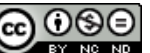

This work is licensed under a Creative Commons Attribution-NonCommercial-NoDerivatives 4.0 International (CC BY-NC-ND 4.0) License. 